\title{
Long-term Impaired Neutrophil Migration in Mice Overexpressing Human Interleukin-8
}

\author{
W. Scott Simonet, * Tamar M. Hughes, ${ }^{*}$ Hung Q. Nguyen, ${ }^{*}$ Lisa D. Trebasky, ${ }^{\ddagger}$ Dimitry M. Danilenko, ${ }^{\$}$ \\ and Eugene S. Medlock ${ }^{\ddagger}$ \\ * Department of Developmental Biology, ${ }^{\ddagger}$ Department of Experimental Hematology, and ${ }^{\S}$ Department of Experimental Pathology, \\ Amgen Center, Thousand Oaks, California 91320
}

\begin{abstract}
The proinflammatory chemokine interleukin-8 (IL-8/NAP1) has been implicated in recruiting neutrophils to sites of acute and chronic tissue inflammation. In transgenic mice, elevated serum IL-8 levels ranging from 1 to $118 \mathrm{ng} / \mathrm{ml}$ were correlated with proportional increases in circulating neutrophils and proportional decreases in L-selectin expression on the surface of blood neutrophils. No change in the expression of the $\beta 2$-integrins Mac-1 and LFA-1 was apparent on peripheral blood neutrophils of the IL-8 transgenic mice. Additionally, L-selectin expression on bone marrow neutrophils and neutrophil precursors was normal in all transgenic lines. IL-8 transgenic mice demonstrated an accumulation of neutrophils in the microcirculation of the lung, liver and spleen. Moreover, there was no evidence of neutrophil extravasation, plasma exudation or tissue damage in any IL-8 transgenic mice. Neutrophil migration into the inflamed peritoneal cavity was severely inhibited in IL8 transgenic mice, but not in nontransgenic littermates. The IL-8 transgenic mice should serve as useful models for studying the putative role of neutrophils in mediating tissue damage in models of inflammation, such as hepatic ischemia and reperfusion injury, cecal puncture and ligation, and glomerulonephritis. (J. Clin. Invest. 1994. 94:1310-1319.) Key words: transgenic mice • leukocyte migration • L-selectin $\cdot$ chemokine $\cdot \boldsymbol{\beta}_{2}$-integrins
\end{abstract}

\section{Introduction}

One important hallmark of acute and chronic inflammatory disease is the migration of leukocytes from the peripheral blood into tissues. In response to infection, tissue accumulation of phagocytic leukocytes is necessary for the elimination of infectious agents such as bacterial and viral pathogens. However, the excessive accumulation and activation of neutrophilic leukocytes in such an inflammatory reaction also can lead to local tissue damage. Such damage is mediated by proteolytic enzymes and free-radical formation resulting from the neutrophilic respiratory burst at the site of infection (1).

The proinflammatory cytokine interleukin 8 /neutrophil-ac-

Address correspondence to W. Scott Simonet, The Amgen Center, 1840 DeHavilland Drive, MS 14-1-B-219, Thousand Oaks, CA 91320.

Received for publication 17 February 1994 and in revised form 18 May 1994.

J. Clin. Invest.

(C) The American Society for Clinical Investigation, Inc.

$0021-9738 / 94 / 09 / 1310 / 10 \$ 2.00$

Volume 94, September 1994, 1310-1319 tivating peptide-1 (IL-8/NAP-1) ${ }^{1}(2-7)$, has been implicated as one important mediator of neutrophil infiltrates identified in the epidermal scales of psoriasis patients $(8,9)$, the synovial fluid of rheumatoid arthritis patients $(10,11)$, the colonic mucosa of individuals with ulcerative colitis $(12,13)$, and in the lung of patients with idiopathic pulmonary fibrosis or adult respiratory distress syndrome $(14,15)$. $\mathrm{IL}-8$ is expressed as a 99 amino acid protein by monocytes, endothelial cells, fibroblasts, and many cell types of epithelial origin (2). Following cleavage of a signal peptide and further proteolytic processing at the amino terminus, $I L-8$ is secreted predominately as an 8.4$\mathrm{kD}$ protein of 72 amino acids by monocytes or an $8.9-\mathrm{kD}$ protein of 77 amino acids by endothelial cells and fibroblasts (16-18). Upon binding to receptors on the surface of neutrophils, IL-8 mediates neutrophil migration into tissues by stimulating rolling neutrophils in the vasculature to adhere to endothelial cells. This adhesive step is presumably caused by IL- 8 mediated shedding of L-selectin and upregulation of $\beta 2$-integrins on the surface of the neutrophils (19-21). IL-8 has also been reported to induce shape changes in neutrophils, activate the respiratory burst, and stimulate release of oxygen free radicals, elastase and other neutral proteases which contribute to the localized tissue damage characteristic of an inflammatory reaction (4). In addition to its proinflammatory role in recruiting neutrophils to local sites of acute and chronic inflammation, IL-8 may have some anti-inflammatory properties. IL- 8 has been reported to inhibit neutrophil adhesion to activated vascular endothelium $(16,17)$, and short-term intravascular delivery of IL-8 to rabbits was found to block neutrophil migration to sites of cytokineinduced inflammation $(22,23)$.

Human IL-8 exhibits variable chemotactic potency for neutrophils of several different species, including monkey, rabbit, dogs, and mice (24). To investigate the long-term in vivo biological effects of IL-8, we tested the ability of human IL-8 to influence blood-borne neutrophil levels, neutrophil surface marker expression, and targeted neutrophil migration in IL-8 transgenic mice. In mice secreting high levels of human IL-8, L-selectin expression is decreased on the neutrophil surface, thioglycollate-mediated neutrophil extravasation is impaired, and neutrophils accumulate in the vascular beds of the lungs, liver, and spleen.

\section{Methods}

Constructs. To target liver expression of transgenes, a 774-bp PstI-XbaI DNA fragment [ which contains the liver specific enhancer or hepatocyte

1. Abbreviations used in this paper: GCSF, granulocyte colony stimulating factor; LAM, leukocyte adhesion molecule; LECAM, leukocyteendothelial cellular adhesion molecule; NAP, neutrophil activating peptide; WBC, white blood cell. 
control region (HCR)] from the human apolipoprotein (apo) C-I/C-I' intergenic region on chromosome 19 (25) was subcloned into the PstI$\mathrm{XbaI}$ sites of pUC19. The resulting clone was designated pCI-CI' PX\#8.

A 1.45-kb KpnI fragment containing a continuous piece of DNA consisting of $650 \mathrm{bp}$ of the human apoE gene 5 '-flanking sequence, the first exon, first intron and a portion of the second exon of the apoE gene was excised from the vector pHE54 (25). This 1.45-kb fragment was inserted into the Kpn-I site of pCI-CI' PX\#8, to generate pHCR$\mathrm{HEP}$. The 2.2-kb enhancer-promoter-intron cassette was excised from pHCR-HEP as a HindIII-EcoRI fragment and cloned into HindIII-EcoRI cut pBSSK $\mathrm{II}^{+}$to generate pHCR-HEP BS(HE).

An SV40 poly A signal was amplified by PCR using the plasmid $\mathrm{V}_{19-10}$ as template. The 242-bp poly A cassette was amplified as a NotISacII fragment. The fragment was sequenced, showing $100 \%$ homology to the template, and was subcloned into NotI-SacII cut pBSSK II ${ }^{+}$, to generate pBS-PA (NS).

The human IL-8 cDNA was used as template to PCR amplify a SpeI-NotI fragment that was subcloned into SpeI-NotI cut pBS-PA (NS) to produce pIL-8 PA. The amplified IL-8 sequence, which lacked a portion of the $3^{\prime}$ untranslated sequence of the CDNA, was sequence verified and found to be $100 \%$ homologous to human IL-8 in the coding region.

Finally, the HCR-apoE promoter-intron cassette was excised from pHCR-HEP BS(HE) as a XhoI-SpeI fragment and subcloned into XhoISpeI cut pIL-8 PA to generate pHCR-HEP IL-8 PA (abbreviated HE8).

To target intestinal expression of transgenes, the PCR amplified (sequence varified) rat intestinal fatty acid binding protein (FABP) promoter in the form of a 1.2-kb EcoRI DNA fragment was cloned into the EcoRI site of pUC19. The human apoE first intron with the adjacent acceptor and donor splice sites was amplified as a KpnI fragment and subcloned into $\mathrm{KpnI}$ cut $\mathrm{FABP}_{\mathrm{TB}}$. The resulting vector was designated FABP-Eintron. The promoter-intron cassette was excised from FABPEintron as an EcoRI fragment and cloned into EcoRI cut pBSSK II $^{+}$ to generate pFE BS. The promoter-intron cassette was subcloned in both orientations for future use. Finally, the promoter-intron cassette was excised from the subclone of $\mathrm{pFE}$ BS with the desired orientation as a XhoI-SpeI fragment and subcloned into Xhol-SpeI cut pIL-8 PA to generate pFE-IL-8 PA (abbreviated FE8).

Preparation and analysis of transgenic mice. For microinjection, the vectors, HE8 and FE8, were digested with XhoI, ScaI, and AflIII, and the 3.3 and 3.1-kb XhoI-AflIII inserts from the respective vectors were purified on a $0.8 \%$ BRL ultrapure DNA agarose gel and diluted to $1 \mu \mathrm{g} / \mathrm{ml}$ in $5 \mathrm{mM}$ Tris, pH 7.4, $0.2 \mathrm{mM}$ EDTA. Single-cell embryos from BDF1 $\times$ BDF1-bred mice were injected essentially as described (26). Embryos were cultured overnight in a $\mathrm{CO}_{2}$ incubator and 15 to 20 two-cell embryos were transferred to the oviducts of pseudopregnant CD1 female mice. Offspring were weaned at 3-4 wk of age and DNA was prepared from a $1-\mathrm{cm}$ portion of their tails. Transgenic founder animals, averaging $15-20 \%$ of the littermates derived from implanted embryos, were identified by PCR using primers which amplified a 368bp fragment of the human apoE first intron. The animals used for analysis were $\mathrm{F} 1$ and $\mathrm{F} 2$ transgenic heterozygous males between 8 and 16 wk of age.

Preparation and analysis of total RNA. Total cellular RNA was isolated from various tissues of transgenic and control mice as described (27). For Northern blot analysis, $20 \mu \mathrm{g}$ of total RNA from the desired tissue was processed and electrophoresed on a $1.0 \%$ formaldehyde gel essentially as described (28). The RNA was transferred to Hybond-N nylon membrane ${ }^{(\mathrm{TM})}$ (Amersham), and probed with ${ }^{32} \mathrm{P}$-labeled cDNA to human IL- 8 or mouse $\beta$-actin. Hybridization was performed overnight at $42^{\circ} \mathrm{C}$ in $50 \%$ formamide, $5 \times$ sodium chloride sodium phosphate EDTA, $0.5 \%$ SDS, $5 \times$ Denhardt's solution, $100 \mu \mathrm{g} / \mathrm{ml}$ denatured salmon sperm DNA and $2-4 \times 10^{6} \mathrm{cpm}$ of labeled probe $/ \mathrm{ml}$ of hybridization buffer. After hybridization, blots were washed twice at room temperature, $5 \mathrm{~min}$ each, in $2 \times \mathrm{SSC}, 0.1 \%$ SDS and twice at $55^{\circ} \mathrm{C}, 5-$ $10 \mathrm{~min}$ each, in $0.1 \times \mathrm{SSC}, 0.1 \%$ SDS. Blots were then exposed to Kodak x-ray film at $-70^{\circ} \mathrm{C}$ for $1-5 \mathrm{~d}$.

Antibody production and Western blot analysis. A rabbit polyclonal antibody to human IL-8 was prepared by subcutaneous injection of three New Zealand white rabbits with unconjugated recombinant human IL8 in Freund's adjuvant. Of several lots tested, rabbit 1912 produced the highest titer antisera, which subsequently proved useful for Western blot analysis of transgenic mouse and control mouse serum. For detection of IL-8 in mouse serum, 1-3 $\mu \mathrm{l}$ of transgenic and nontransgenic mouse serum was electrophoresed on a $16 \%$ polyacrylamide gel. Purified endothelial form of IL-8 (77 amino acid form) was included as a standard and migration control. Following electrophoresis in a Novex mini-gel apparatus, the fractionated mouse proteins were electroblotted onto $\mathrm{Hy}$ bond-ECL ${ }^{\mathrm{TM}}$ nitrocellulose (Amersham Intl., Buckingham, England). Western blotting was performed as described in the ECL Western blotting protocol manual supplied with the Amersham kit, but with the following variations. Initial blocking of the filters was performed using $10 \%$ dried milk in PBS with $0.1 \%$ Tween-20 ${ }^{\mathrm{TM}}$ (Atlas Chemical Co., Miami, FL). Incubation of primary antibody (1912) was performed at a dilution of 1:2000 for $45 \mathrm{~min}$ at room temperature. An HRP labeled anti-rabbit IgG from donkey (1:7500 dilution) was used as the second antibody.

ELISA of Human IL-8. Quantitation of serum IL-8 levels in transgenic and nontransgenic $\mathrm{F} 1$ heterozygous offspring from different lines was performed using an ELISA kit (Biosource International, Camarillo, CA) to human IL-8 as described in the protocol accompanying the kit.

Circulating white blood cell analysis. Circulating white blood cells (WBC) were counted in a 1:500 dilution of freshly prepared whole blood on a Sysmex F-800 blood cell counter (Toa Medical Electronics Co., Ltd., Kobe, Japan), following lysis of the red blood cells with Quicklyser $^{\text {TM }}$ (Toa Medical Electronics Co., Ltd). Differential leukocyte analysis was done using $3 \mu \mathrm{l}$ of whole blood spread on a glass slide and subjected to Wright's-Giemsa staining. At least 100 cells were counted from each slide by visualizing under a $100 \times$ oil immersion lens on an Olympus $\mathrm{CH} 2$ microscope. For each transgenic line reported, a minimum of five individual F1 heterozygotes were analyzed. Quantitation of neutrophil levels was determined by multiplying the percentage of neutrophils by the total WBC count obtained from the Sysmex counter.

Immunohistochemistry of animal tissues. Tissues were fixed overnight in 10\% neutral buffered zinc formalin (Anatech, Battle Creek, MI), paraffin embedded, and sectioned at $3 \mu \mathrm{m}$. To highlight neutrophils in tissues, sections were incubated with a 1:300 dilution of rabbit antisera directed at human myeloperoxidase (Dako Corp., Carpinteria, CA), then incubated with a 1:500 dilution of biotinylated goat antisera directed at rabbit IgG (H + L chains) (Vector Labs, Inc., Burlingame, CA), and finally incubated with a 1:100 dilution of an avidin-biotin complex conjugated to horseradish peroxidase (Vectastain Elite; Vector Labs, Inc.). Specific immunohistochemical staining was detected using diaminobenzidine (DAB; Sigma Co., St. Louis, MO) as the enzyme substrate. All sections were blocked with $10 \%$ normal goat serum prior to incubation with the primary antibody.

FACS analysis of whole blood and bone marrow. Blood was collected into EDTA tubes. Femurs were harvested and flushed with phosphate buffered saline supplemented with $2 \%$ heat inactivated fetal bovine serum (PBS/FBS). $30 \mu \mathrm{l}$ of blood or $10^{6}$ bone marrow cells were incubated with $20 \mu \mathrm{l}$ of antibody for $30 \mathrm{~min}$ at $4^{\circ} \mathrm{C}$. Cells were washed with PBS/FBS and red blood cells were lysed with FACS lysing solution (Becton Dickinson Vacutainer Systems, Rutherford, NJ). Cells were washed and analyzed using a FACScan cell analyzer (Becton Dickinson Vacutainer Systems). Monoclonal antibodies purchased from Pharmingen were used: Lecam-1 (MEL-14), LFA-1 (CD11a, clone 2D7), Gr-1(RB6-8C5), Mac-1(M1/70), B-220 (RA3-6B2), Thy 1.2 (53$2.1)$ and Rat Ig controls. All antibodies were directly conjugated with either fluorescein isothiocyanate (FITC) or phycoerythrin (PE).

IL-8 and thioglycollate-mediated neutrophil migration. 8-12-wkold male transgenic and nontransgenic F1 littermates of the HE8 51 line were challenged with intraperitoneal injections of recombinant human IL-8 ( 77 amino acid form, endotoxin level $<2.5 \mathrm{EU} / \mathrm{ml}$ ), thioglycollate broth, or PBS. Mice were killed $4 \mathrm{~h}$ after IL-8 injection and 1-4 h after 
Figure 1. Design of constructs for expression of human IL-8 in transgenic mice. The human apoE promoter, its associated liver specific enhancer (HCR) and the rat fatty acid binding protein (FABP) promoter are described in Methods. The HE8 construct was designed for hepatic expression of human interleukin 8 . The FE8 construct was designed to direct expression of human interleukin 8 in intestinal villus enterocytes. For the HE8 construct, 9 of 52 potential offspring had the transgene integrated into their genome. For the HE8 construct, 11 of 56 potential offspring harbored the transgene in their genome. Analysis were performed on F1 transgenic heterozygotes from individual lines.

thioglycollate injection and neutrophils in the peritoneal exudate were quantitated.

\section{Results}

Generation and analysis of IL-8 transgenic mice. To generate mice in which neutrophil migration might be influenced by establishing or disrupting gradients of neutrophil chemoattractants, the human neutrophil chemotactic cytokine IL-8 was expressed in either the liver or the gastrointestinal tract of transgenic mice.

To direct liver expression of human IL-8, we used the liver specific enhancer (HCR) and human apoE promoter from the apoE/C-I gene locus on human chromosome 19 (Fig. 1, HE8) $(25,29)$. This enhancer-promoter combination had been shown previously to direct high level expression of the linked human apoE and apoC-I genes in hepatocytes of transgenic mice (25). A second transgene construct (Fig. 1, FE8), designed to direct IL-8 expression to the gastrointestinal tract, used a rat fatty acid binding protein promoter previously shown to direct lineage-specific expression of transgenes to the villus enterocytes of the small intestine and proximal colon of mice (30, 31 ). The same human apoE first intron was used in both constructs because of previous reports that introns improve expression of transgenes in mice $(26,32,33)$.

Five independent lines harboring the HE8 transgene were analyzed for expression of human IL-8 RNA by Northern blot analysis with a cDNA probe to human IL-8 (Fig. 2 a). Heterozygous F1 offspring from lines 7, 26, and 51 had high levels of IL-8 mRNA in the liver, with much lower levels detectable in the kidney. The human apoE promoter has been reported previously to direct kidney expression of transgenes, provided that the appropriate regulatory elements are present $(25,34)$, thus it was not surprising to find low levels of kidney expression. No IL-8 mRNA was detected in other tissues analyzed (data not shown). HE8 line 36 exhibited markedly lower levels (100fold) of expression in the liver when compared to the three high expressing lines. Line 49 was a nonexpressing line and no hybridizable signal was detected when RNA prepared from a nontransgenic mouse was analyzed.

Heterozygous F1 offspring from six lines for the FE8 construct expressed IL-8 mRNA in the small intestine. The expression pattern of four of these lines is shown in Fig. $2 b$. Lines 13 and 54 had relatively high expression compared to lines 44 , 6 (Fig. $2 b$ ), and two other lines which are not shown. Lines
$13,54,44$, and 6 expressed IL-8 mRNA at highest levels in the jejunum, with lower levels in ileum and duodenum. No expression was detected in the liver (Fig. $2 b$ ). Mouse $\beta$-actin RNA was probed as a control for RNA loading. Liver typically gave a lower signal for mouse $\beta$-actin RNA compared to other tissues.

Analysis of serum from the HE8 transgenic founder mice by Western blot analysis with a rabbit polyclonal antibody to human IL-8 revealed abundant levels of the transgene product in the blood of mice from lines 7, 26, and 51 (Fig. 2 c). A strong correlation between RNA expression in the liver and circulating levels of IL-8 was observed. Of the intestinal expressing transgenics, the FE8 13 founder had the highest serum levels of IL-8 (Fig. $2 c$ ). The IL-8 protein detected in all transgenic lines, whether of liver or intestinal origin, migrated in SDS at a position close to the 77 amino acid endothelial cellderived IL-8 standard.

Correlation of serum IL-8 levels with circulating neutrophil numbers. Serum was analyzed from a minimum of five transgenic $\mathrm{F} 1$ heterozygous offspring from each expressing line for circulating human IL-8 using a specific ELISA (Table I and additional data not shown). Circulating white blood cell analysis of the same mice revealed that total white blood cell levels and absolute neutrophil numbers were markedly elevated in the highest HE8 expressing lines, with more modest increases observed in lower expressing lines. Among a total of seven independent lines of transgenic mice analyzed, a strong correlation was observed between serum IL-8 levels and absolute numbers of circulating neutrophils. Elevated blood neutrophils were found in mice with steady state serum IL-8 levels as low as 1.2 $\mathrm{ng} / \mathrm{ml}$ and as high as $118 \mathrm{ng} / \mathrm{ml}$. Representative results from three different transgenic lines with steady state serum IL-8 levels at three different orders of magnitude are shown in Table I.

Neutrophils in the microcirculation of vascularized tissues. Histological analysis of myeloperoxidase-stained tissues of high and low expressing transgenic animals for either the HE8 or FE8 constructs revealed increased neutrophil accumulation in the microcirculation of liver, lung, and spleen of high expressing animals compared to nontransgenic littermates (Fig. 3). While neutrophil accumulation in the capillaries and sinuses of highly vascularized tissues was observed, no extravascular neutrophilic infiltrates were observed in any tissue analyzed, including those expressing the transgene product. In addition, no plasma exudation (as accessed by measuring total protein levels in bronchoalveolar lavage fluid of transgenic and control mice) or tissue damage was evident in any of the transgenic mice, even in high expressing lines in which increased numbers of neutrophils were observed in the circulation for as long as $12 \mathrm{mo}$. High resolution microscopy revealed that neutrophils were marginated within postcapillary venules (Fig. 3, inset).

Cytofluorimetric analysis of $L$-selectin and $\beta 2$-integrin expression on neutrophils. FACS analysis using lineage-specific, cell surface marker antibodies revealed that peripheral blood $\mathrm{Gr}-1^{+}$cells (an antigenic determinant specific for neutrophils) were markedly elevated in the transgenic mice compared to their nontransgenic littermates (Fig. 4). There was no significant difference in the relative numbers of circulating lymphocytes, as measured by B-220 ${ }^{+}$and Thy $1.2^{+}$cells in the blood of transgenic vs. nontransgenic mice (data not shown). Similar analysis of bone marrow from the same mice revealed normal bone marrow cellularity, with no significant change in either 

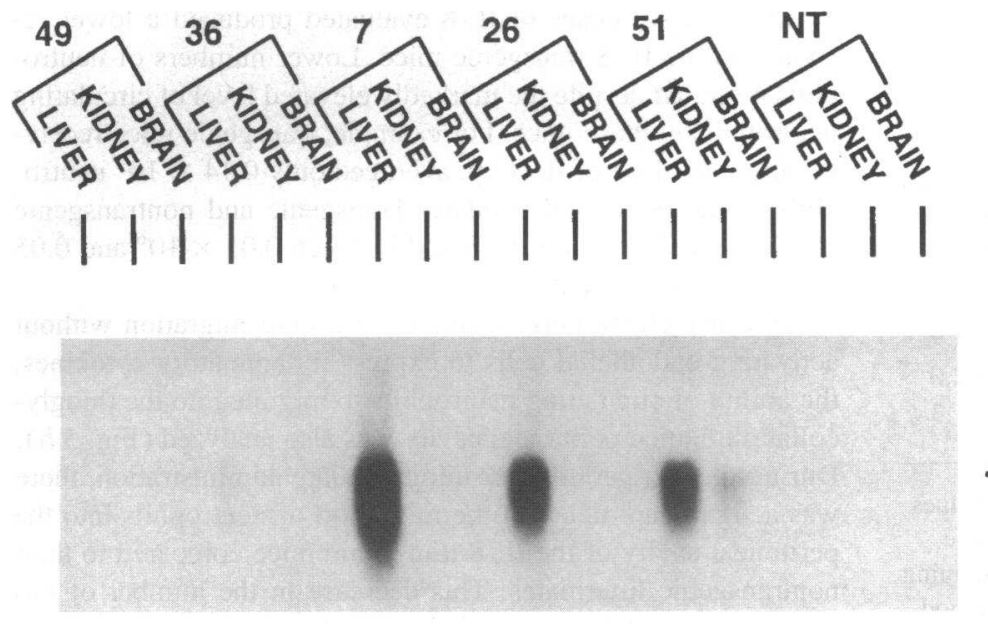

- IL-8

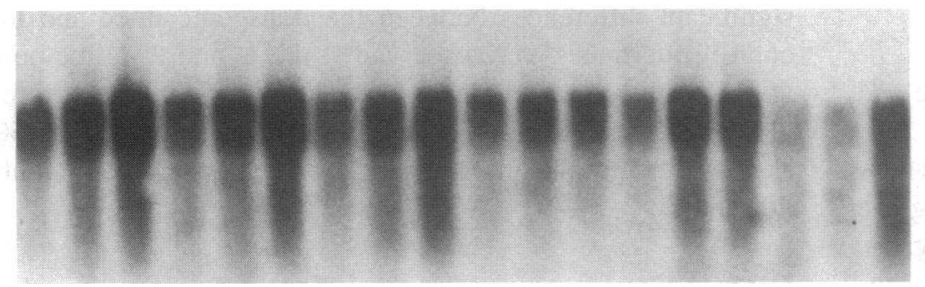

\section{$-\beta-A C T I N$}

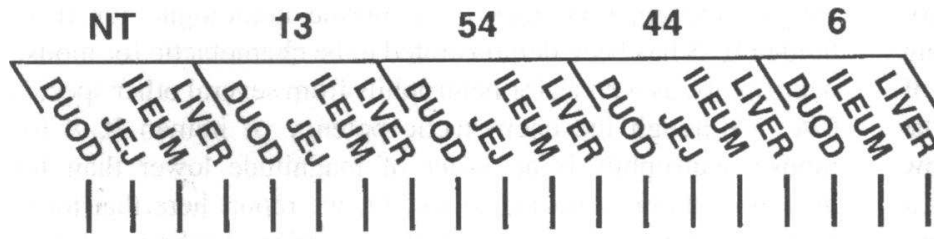

b
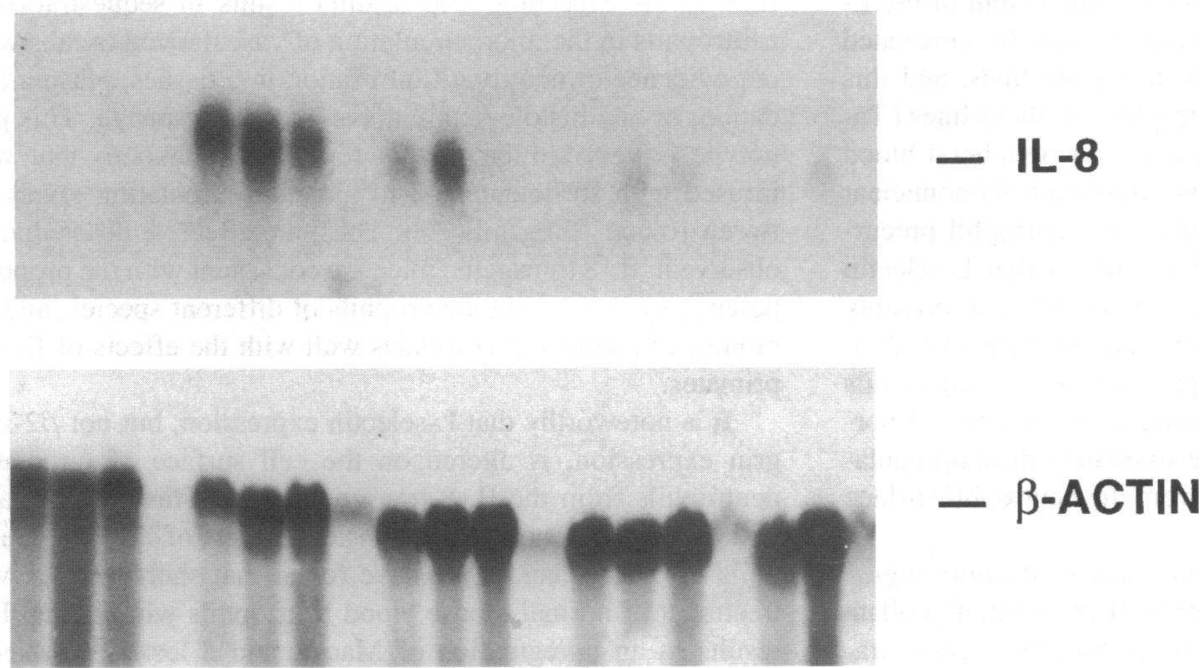

Figure 2. Northern blot and Western blot analysis of human IL-8 expression in transgenic mice. ( $a$ ) Northern blot analysis of human IL-8 RNA expression in HE8 transgenic mouse tissues. F1 heterozygotes from five separate transgenic lines (designated 49, $36,7,26$, and 51 ) and one nontransgenic control (NT) were analyzed for expression of the IL-8 transgene and endogenous mouse $\beta$ actin gene in the liver, kidney, and brain. ( $b$ ) Northern blot analysis of human IL-8 RNA expression in FE8 transgenic mouse tissues. F1 heterozygotes from four separate transgenic lines (designated 13, 54, 44, and 6) and one nontransgenic control (NT) were analyzed for expression of the IL-8 transgene and endogenous mouse $\beta$-actin gene in the liver and different regions of the small intestine. (c) Western blot analysis of human IL-8 in transgenic and nontransgenic mouse serum. Serum from the founder animals of eight HE8 lines and the highest expressing FE8 line, \#13, were analyzed for the presence of human IL-8 as described in Methods. Purified endothelial form

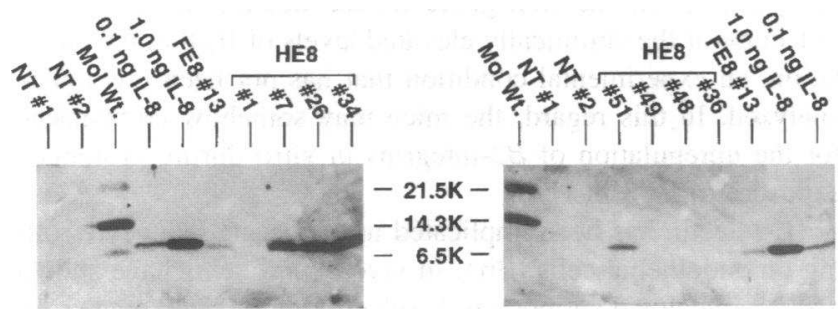

C ( 77 amino acid) of IL-8 was run as a positive control on the gels and nontransgenic mouse serum (NT) was analyzed as a negative control. HE8 founder \#34 failed to generate any progeny, and HE8 founder \#26 had the transgene integrated at two different chromosomal locations which segregated in subsequent generations. 
Table I. Correlation of Serum IL-8 Levels with Circulating Neutrophils and L-selectin Staining Intensity on the Surface of Peripheral Blood Neutrophils

\begin{tabular}{|c|c|c|c|c|}
\hline Line* & Serum IL-8 & Total leukocytes & Neutrophil counts & L-selectin ${ }^{8}$ \\
\hline & $n g / m l$ & $\times 10^{-3} / \mu l$ & $\# / \mu l$ & $\begin{array}{l}\text { mean fluorescent } \\
\text { channel }\end{array}$ \\
\hline $\mathrm{NT}^{\ddagger}$ & $\mathrm{ND}^{\ddagger}$ & $8.1 \pm 3.4$ & $841 \pm 308$ & $17.3 \pm 2.9$ \\
\hline HE8 36 & $1.2 \pm 0.3$ & $11.1 \pm 3.2$ & $1901 \pm 704$ & $11.4 \pm 1.9$ \\
\hline FE8 13 & $15.2 \pm 4.5$ & $10.5 \pm 2.8$ & $3578 \pm 1215$ & $7.6 \pm 2.0$ \\
\hline HE8 51 & $106.3 \pm 18.6$ & $16.8 \pm 2.7$ & $6110 \pm 1700$ & $6.7 \pm 1.3$ \\
\hline
\end{tabular}

* F1 transgenic heterozygotes were analyzed for each line. All values are mean and standard deviation of at least 5 animals for each line. ${ }^{\ddagger} \mathrm{NT}$, nontransgenic; ND, not detectable. ${ }^{8} \mathrm{~V}$ Values for L-selectin staining are the mean fluorescent channel for each population of $\mathrm{Gr}-1^{+}$blood neutrophils.

$\mathrm{Gr}-1^{+}, \mathrm{B}-220^{+}$, or Thy $1.2^{+}$cells in $\mathrm{IL}-8$ transgenic mice and littermate controls (data not shown).

Analysis of surface marker expression on Gr-1 positive neutrophils revealed that the circulating neutrophils in transgenic offspring of the HE8 36 and 51 lines expressed the $\beta 2$-integrins, LFA-1, and Mac-1 (Fig. 4). While there was a significant increase in Gr-1 positive cells, no significant change in surface expression of the $\beta 2$-integrin molecules was detected on neutrophils from either the high expressing (line 51 ) or low expressing (line 36) HE8 transgenic mice (Fig. 4). However, analysis of L-selectin (also known as LECAM-1, Leu8, LAM-1, or the MEL14 antigen) levels on the neutrophils of the high and low expressing transgenic mice, revealed a significant loss of Lselectin expression on peripheral blood neutrophils when compared to nontransgenic controls (Table I). The extent of the Lselectin decrease on circulating neutrophils directly correlated with the level of expression of IL-8 in several lines, and this decrease paralleled the observed neutrophilia in these lines ( Table I). The loss of L-selectin was specific for peripheral blood neutrophils and was not observed on circulating mononuclear leukocytes or bone marrow neutrophils and neutrophil precursors (data not shown). These findings suggest that L-selectin is lost from neutrophils upon entering the circulation, possibly by proteolytic shedding from the neutrophil surface (19-21). The apparent lack of $\beta_{2}$-integrin upregulation on neutrophils from the transgenic mice was surprising, as incubation of normal mouse blood with IL-8 ex vivo resulted in both an upregulation of Mac-1 and loss of L-selectin from the neutrophil surface (data not shown).

Effects of intravascular IL-8 on targeted neutrophil migration. We tested the ability of exogenous IL- 8 or thioglycollate (a potent mediator of inflammation) to mediate targeted migration of neutrophils into the peritoneal cavity of transgenic and nontransgenic mice. The IL-8 transgenic mice and their nontransgenic littermates were challenged for their response to "endotoxin-free" recombinant human IL-8. Doses of IL-8 ranging from 0.1 to $1 \mu \mathrm{g}$ were injected intraperitoneally and found to stimulate a dose-dependent infiltration of neutrophils into the peritoneal cavity of nontransgenic mice (Fig. $5 a$ ). $4 \mathrm{~h}$ after administration of $0.1 \mu \mathrm{g}$ of IL-8, the number of neutrophils in the peritoneal cavity of the nontransgenic mice averaged 0.22 $\times 10^{6}$. This number increased to $1.05 \times 10^{6}$ with a dose of 1 $\mu \mathrm{g}$ of IL-8. All doses of IL-8 evaluated produced a lower response in the IL-8 transgenic mice. Lower numbers of neutrophils migrated despite the markedly elevated level of circulating neutrophils in these mice. For example, transgenic mice receiving the $1 \mu \mathrm{g}$ dose of IL-8 i.p. averaged only $0.14 \times 10^{6}$ neutrophils in the peritoneal exudate. Transgenic and nontransgenic mice that received only PBS had between $0.01 \times 10^{6}$ and 0.05 $\times 10^{6}$ peritoneal neutrophils.

Because chemokines mediate neutrophil migration without activating endothelial cells to express inflammatory cytokines, the ability of circulating neutrophils to migrate into the thioglycollate-inflamed peritoneal cavity was also analyzed (Fig. $5 b$ ). During the 4-h period after thioglycollate administration, there was a significant delay in the migration of neutrophils into the peritoneal cavity of the IL- 8 transgenic mice compared to their nontransgenic littermates. This decrease in the number of migrating neutrophils occurred despite the 8- to 10-fold higher level of circulating neutrophils in the transgenic mice. The most significant differences between the transgenic mice and their control littermates were observed at the 1- and 2-h time points after thioglycollate administration. However, by $4 \mathrm{~h}$ after thioglycollate administration, the transgenic mice had about $50 \%$ as many neutrophils in the peritoneal cavity as their control littermates.

\section{Discussion}

Despite the apparent lack of a murine homologue for $\mathrm{IL}-8$, human IL-8 has been demonstrated to be chemotactic for mouse neutrophils, as well as for neutrophils from several other species (24). Although the chemotactic potency of human $\mathrm{IL}-8$ for mouse neutrophils is an order of magnitude lower than for human or monkey neutrophils (24), we report here that longterm overexpression of human IL-8 in mice (leading to serum IL-8 levels exceeding $100 \mathrm{ng} / \mathrm{ml}$ ) results in sequestration of neutrophils in the microcirculation of vascularized organs without evidence of neutrophil infiltration into tissues, plasma exudation, or any histologically apparent tissue damage. This phenotype is very similar to that reported in baboons that were infused with sufficient IL-8 to maintain circulating levels between 10 and $15 \mathrm{ng} / \mathrm{ml}$ (35). Thus, the effective doses that we observe in IL-8 transgenic mice are consistent with the proposed potency of IL-8 on the neutrophils of different species, and the biological phenotype correlates well with the effects of IL-8 in primates.

It is noteworthy that $\mathrm{L}$-selectin expression, but not $\beta 2$-integrin expression, is altered on the cell surface of circulating neutrophils from the IL-8 transgenic mice. This is at variance with previous reports of the transient effects of IL-8 on neutrophils of other species. We have found that short-term ex vivo treatment of normal mouse blood neutrophils with human IL-8 results in an upregulation of Mac-1, and a loss of L-selectin from the neutrophil surface. The absence of any $\beta 2$-integrin upregulation on the transgenic mouse neutrophils may be a reflection of the chronically elevated levels of IL-8 in the circulation, an experimental condition that has not been previously analyzed. In this regard, the mice may somehow compensate for the upregulation of $\beta 2$-integrins in vitro during prolonged exposure to $\mathrm{IL-8}$ in vivo.

L-selectin has been implicated in mediating leukocyte rolling on endothelial cells (36). In vivo experiments have shown that a monoclonal antibody to L-selectin inhibits leukocyte roll- 


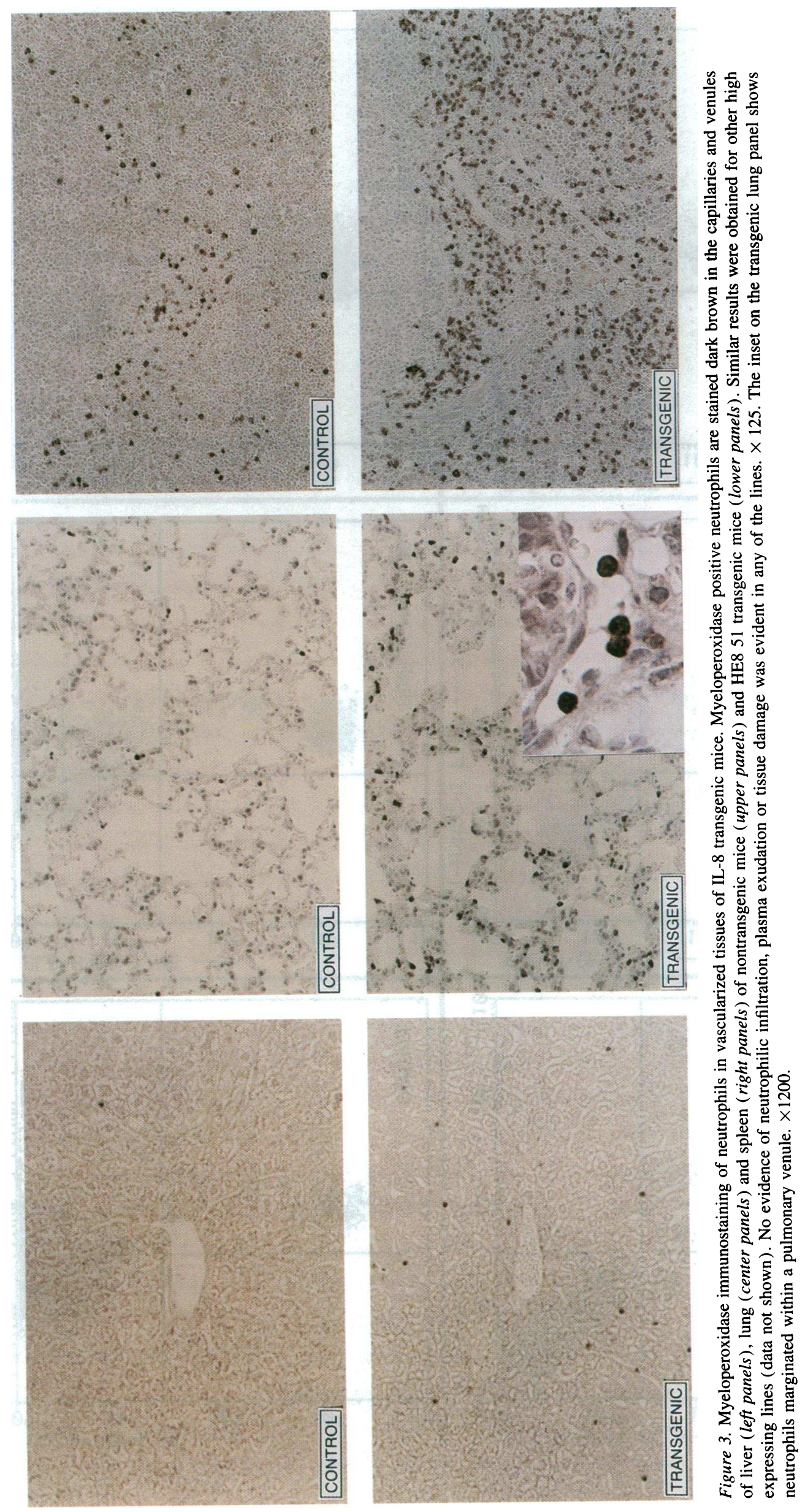




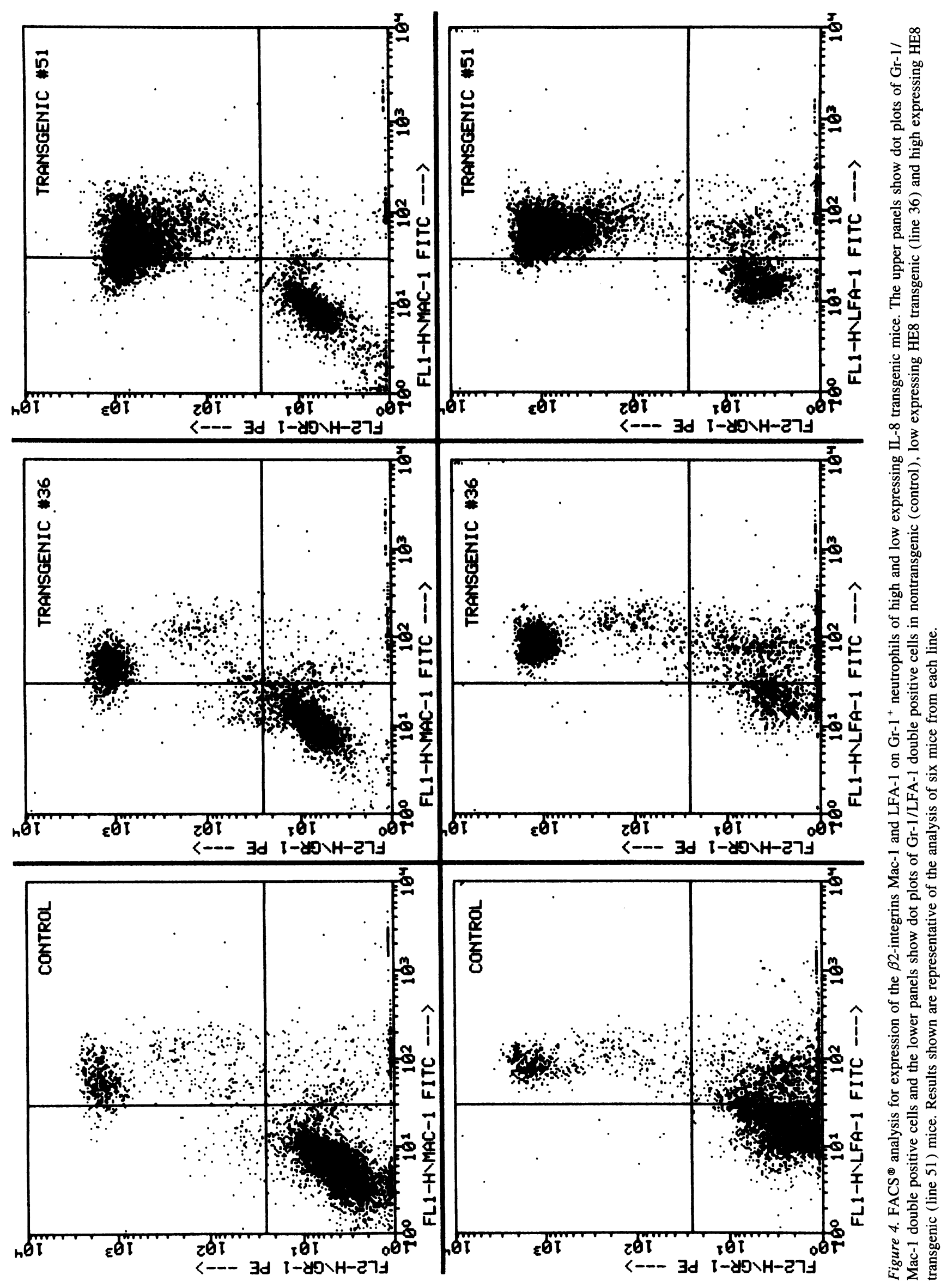



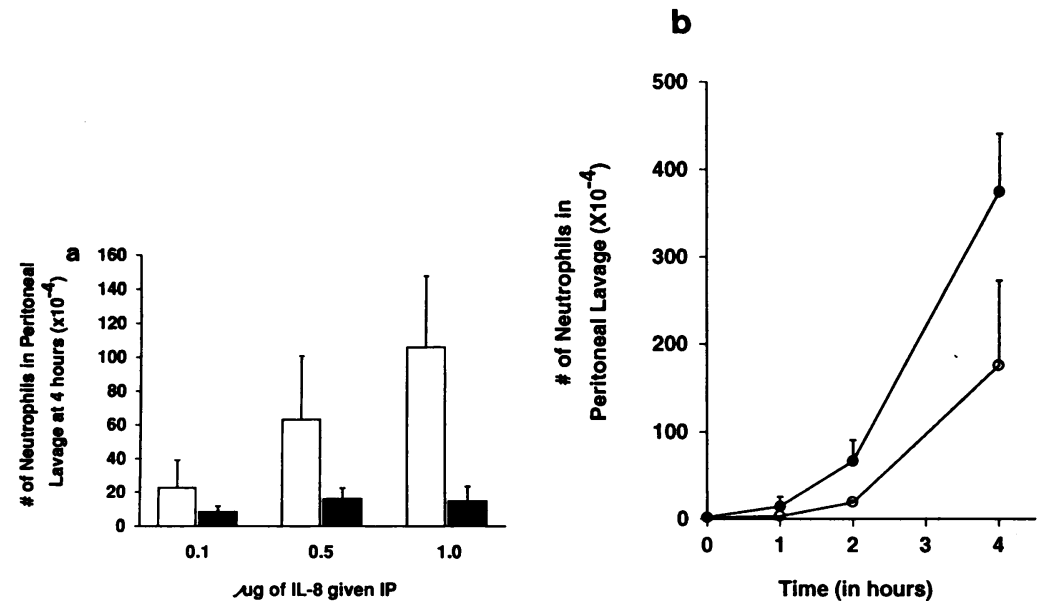

Figure 5. Impaired neutrophil migration in IL-8 transgenic mice. (a) Transgenic and nontransgenic littermates of the HE8 51 line were challenged with intraperitoneal injections of IL-8 (endotoxin $<2.5 \mathrm{EU} / \mathrm{ml}$ ). $4 \mathrm{~h}$ following doses of $0.1,0.5$, or $1.0 \mu \mathrm{g}$, the mice were killed, their peritoneal cavity was lavaged and the number of neutrophils in the peritoneal exudate was counted. Four nontransgenic mice (open boxes) and four transgenic mice (dark boxes) were analyzed at each dose. (b) Transgenic and nontransgenic littermates of the HE8 51 line were challenged with a 1-ml intraperitoneal injection of thioglycollate broth. $0-4 \mathrm{~h}$ after injection, the mice were killed and peritoneal neutrophils were counted. Five nontransgenic (dark circles) and five transgenic (open circles) mice were analyzed at $0,1,2$, and $4 \mathrm{~h}$ after thioglycollate administration.

ing along the vascular wall of rabbit mesenteric venules, supporting an important role for L-selectin in leukocyte-endothelial interaction (36). Neutrophil chemoattractants, including IL-8, have been shown to induce L-selectin shedding from activated mouse neutrophils in vitro (37), and the capacity of activated (L-selectin ${ }^{\text {low }}$ ) neutrophils to localize to the thioglycollate-inflamed peritoneal cavity is decreased compared to unactivated (L-selectin $^{\text {high }}$ ) neutrophils (38). The loss of L-selectin from the peripheral blood neutrophils of IL-8 transgenic mice provides direct in vivo evidence that chemokines can modulate the long-term expression of $\mathrm{L}$-selectin on the surface of circulating neutrophils. This loss of L-selectin occurred without long-term modulation of $\beta$-integrin expression. Furthermore, no change in bone marrow cellular architecture was evident in any of the transgenic lines. Our results demonstrate detectable decreases in surface L-selectin expression on circulating neutrophils at steady state IL-8 levels as low as $1.2 \mathrm{ng} / \mathrm{ml}$ in mouse serum. Significantly lower levels of L-selectin were detected on circulating neutrophils in mice with higher steady state IL-8 levels. However, even at serum IL-8 levels exceeding $100 \mathrm{ng} / \mathrm{ml}$, no decrease in L-selectin levels on bone marrow neutrophilic precursors was detected.

The impaired neutrophil migration observed in the IL-8 transgenic mice is in agreement with the previously reported effects of intravascular IL- 8 as an inhibitor of PMN accumulation at sites of acute inflammation (22). In this previous study, intravascular administration of IL-8 as a single injection blocked cytokine-induced neutrophil migration into the skin of New Zealand white rabbits over the 2 -h period after the IL-8 injection. The pronounced granulocytosis observed in high expressing IL-8 transgenic mice is likely attributable, at least in part, to the effects of IL-8 on L-selectin expression on the neutrophil surface, rather than effects on endothelial cell surface adhesion molecules, since previous studies have revealed no effects of IL-8 on endothelial adhesion molecule expression (39). Furthermore, the kinetics of neutrophil migration into the thioglycollate-inflamed peritoneal cavity of IL-8 transgenic mice closely resembles that previously reported when a soluble murine L-selectin-IgG chimera or L-selectin antibody was used to block neutrophil migration (37).

Despite these considerations, other effects of IL- 8 may also contribute to the impaired neutrophil migration observed in the IL-8 transgenic mice. Recently, the decreased in vivo migratory capacity of rabbit neutrophils following intravenous delivery of
IL-8 was reported to occur without significant loss of L-selectin from the neutrophil surface (23). Although this study involved a single intravenous dose of IL-8, which only transiently elevated serum IL-8 levels for $30 \mathrm{~min}$ after the injection, these results suggest that IL-8 may inhibit neutrophil migration by L-selectin-independent mechanisms as well. In this regard, interleukin 8 and other chemoattractants that exhibit a leukocyte adhesion inhibition (LAI) effect have been demonstrated to cause rapid and profound changes in the conformation of actin microfilaments in neutrophils (39). This IL-8 induced actin polymerization may result in the inability of neutrophils to form intercellular contacts necessary for adhesion and subsequent migration across the endothelium.

Additionally, chronic high levels of IL-8 in serum may render circulating neutrophils unresponsive to chemotaxis signals by downregulating putative murine chemokine receptors, or by effectively disrupting endogenous gradients of chemoattractant molecules, rather than establishing chemotactic gradients similar to those generated in an acute, localized inflammatory reaction. However, if gradients of IL-8 are the main determinant of neutrophil migration, then an intraperitoneal bolus of IL- 8 given to the transgenic mice would have been expected to overcome the high serum levels and result in targeted neutrophil migration into the peritoneal cavity. This was clearly not the case, as IL8 mediated neutrophil migration in the transgenic mice was severely impaired at several different doses of IL- 8 when compared to their nontransgenic littermates.

In preliminary experiments, we were unable to detect changes in granulocyte colony stimulating factor (GCSF) activity when serum from the transgenic mice and control mice was assayed in a GCSF specific 32-D cell proliferation assay. Thus, increased neutrophil production as a result of cytokine stimulation is not likely to be a contributing factor in the observed granulocytosis in the IL- 8 transgenic mice.

Compromised neutrophil migration due to impaired leukocyte rolling has recently been described in P-selectin-deficient mice (40). In homozygous P-selectin knockout mice, the rate of migration into the peritoneal cavity following intraperitoneal injection of thioglycollate, was significantly decreased compared to wild type mice, which mirrored similar data shown here with intraperitoneal injection of thioglycollate or IL-8 in our IL-8 transgenic mice. However, the phenotype of the Pselectin deficient mice appeared far more subtle than that observed in the transgenic lines overexpressing IL-8. The loss of 
P-selectin expression resulted in an approximate twofold increase in circulating neutrophil levels, without any significant change in absolute peripheral WBC levels. The highest IL-8 expressing lines (HE8 7, 26, and 51 ) had circulating neutrophil levels between 8- and 12-fold higher than their nontransgenic littermates. Moreover, as a result, the absolute circulating WBC levels were increased two- to threefold. These findings may imply distinct roles for L-selectin and P-selectin in mediating rolling of neutrophils on the endothelial surface. L-selectin may play an important role in the initial leukocyte-endothelial interaction as neutrophils begin rolling on the endothelium, whereas endothelial P-selectin may facilitate the rolling of neutrophils only after they have encountered the endothelial cell surface. Alternatively, the more pronounced granulocytosis in IL-8 transgenic mice compared to P-selectin-deficient mice may be a reflection of additional non-L-selectin-dependent mechanisms by which IL-8 inhibits neutrophil adhesion to, and transmigration across, the vascular endothelium. It will be interesting to see if L-selectin-deficient mice exhibit a more pronounced phenotype than was reported about the P-selectin knock out mice.

In summary, we have reported that transgenic mice overexpressing IL-8 exhibit a marked circulating neutrophilia and a decreased neutrophilic exudation into body cavities in response to acute inflammatory stimulants. These IL-8 transgenic mice should serve as useful models for studying the putative role of neutrophils in mediating tissue damage in various models of inflammation, such as hepatic ischemia and reperfusion injury, cecal puncture and ligation, and glomerulonephritis.

\section{Acknowledgments}

The authors thank Michael P. Bevilacqua, Glenn Pierce, and Thomas $R$. Ulich for thoughtful discussions and critical review of the manuscript. Thanks to Babru Samal for IL-8 cDNA. We thank Susan Watson (Genentech) for generously donating thioglycollate broth. Thanks to Tanya Kernodle and Kathy Klopchin for help with microinjections and colony maintainance. Special thanks to John M. Taylor for generous contribution of the rFABP promoter, and Edward Shatzen and Fred Lott for assistance and advice on endotoxin delivery.

\section{References}

1. Chernyak, L., and A. I. Tauber. 1988. The birth of immunology: Metchnikoff, the embryologist. Cell Immunol. 117:218-233.

2. Baggiolini, M., B. Dewald, and A. Walz. 1992. Interleukin-8 and related chemotactic cytokines. In Inflammation: Basic Principles and Clinical Correlates, J. I. Gallin, I. M. Goldstein, and R. Snyderman, editors. Raven Press, New York, 247-264.

3. Furuta, R., J. Yamagishi, H. Kotani, F. Sakamoto, T. Fukui, Y. Matsui, Y. Sohmura, M. Yamada, T. Yoshura, C. G. Larsen, J. J. Oppenheim, and K. Matshshima. 1989. Production and characterization of recombinant human neutrophil chemotactic factor. J. Biochem. 106:436-441.

4. Matsushima, K., and J. J. Oppenheim. 1989. Interleukin 8 and MCAF: Novel inflammatory cytokines inducible by IL-1 and TNF. Cytokine. 1:2-13.

5. Oppenheim, J. J., C. O. C. Zachariae, N. Mukaida, and K. Matsushima. 1991. Properties of the novel proinflammatory supergene "intercrine" cytokine family. Annu. Rev. Immunol. 9:617-648.

6. Rot, A. 1992. Endothelial cell binding of NAP-1/IL-8: role in neutrophil emigration. Immunol. Today. 13:291-294.

7. Yoshimura, T., K. Matsushima, J. J. Oppenheim, and E. J. Leonard. 1987. Neutrophil chemotactic factor produced by lipopolysaccharide (LPS)-stimulated human blood mononuclear leukocytes: partial characterization and separation from interleukin-1 (IL-1). J. Immunol. 139:788-793.

8. Baggiolini, M., S. Walz, and S. L. Kunkel. 1989. Neutrophil-activating peptide-1/interleukin-8, a novel cytokine that activates neutrophils. J. Clin. Invest. 84:1045-1049.

9. Sticherling, M., E. Bornscheuer, J.-M. Schröder, and E. Christophers. 1991.
Localization of neutrophil-activating peptide 1 /interleukin-8 immunoreactivity in normal and psoriatic skin. J. Invest. Dermatol. 96:26-30.

10. Brennan, F. M., C. O. Zachariae, D. Chantry, C. G. Larsen, M. Turner, R. N. Maini, K. Matsushima, and M. Feldmann. 1990. Detection of Interleukin8 biological activity in synovial fluids from patients with rheumatoid arthritis and production of interleukin 8 mRNA by isolated synovial cells. Eur. J. Immunol. 20:2141-2144.

11. Seitz, M., B. Dewald, N. Gerber, and M. Baggiolini. 1991. Enhanced production of neutrophil-activating peptide 1 /interleukin- 8 in rheumatoid arthritis. J. Clin. Invest. 87:63-469.

12. Izzo, R. S., K. Witkon, A. I. Chen, C. Hadjiyane, M. I. Weinstein, and C. Pellecchia. 1992. Interleukin-8 and neutrophil markers in colonic mucosa from patients with ulcerative colitis. Am. J. Gastroenterol. 87:1447-1452.

13. Mahida, Y. R., M. Ceska, F. Effenberger, L. Kurlak, I. Lindley, and C. J. Hawkey. 1992. Enhanced synthesis of neutrophil-activating peptide-1/interleukin-8 in active ulcerative colitis. Clin. Sci. (Lond.). 82:273-275.

14. Chollet-Martin, S., P. Montravers, C. Gibert, C. Elbim, J. M. Desmonts, J. Y. Fagon, and M. A. Gougerot-Pocidalo. 1993. High levels of Interleukin-8 in the blood and alveolar spaces of patients with pneumonia and adult respiratory distress syndrome. Infect. Immun. 61:4553-4559.

15. Hunninghake, G. W., J. E. Gadek, T. J. Lawley, and R. G. Crystal. 1981. Mechanisms of neutrophil accumulation in the lungs of patients with idiopathic pulmonary fibrosis. J. Clin. Invest. 68:259-269.

16. Gimbrone, M. A. Jr., M. S. Obin, A. F. Brock, E. A. Luis, P. E. Hass, C. A. Hebert, Y. K. Yip, D. W. Leung, D. G. Lowe, and W. J. Kohr. 1989 Endothelial interleukin-8: a novel inhibitor of leukocyte-endothelial interactions. Science (Wash. DC). 246:1601-1603.

17. Hebert, C. A., F. W. Luscinskas, J. M. Kiely, E. A. Luis, W. C. Darbonne, G. L. Bennett, C. C. Liu, M. S. Obin, M. A. Gimbrone, Jr., and J. B. Baker. 1990. Endothelial and leukocyte forms of IL-8. Conversion by thrombin and interactions with neutrophils. J. Immunol. 145:3033-3040.

18. Schröder, J. M., M. Sticherling, H. H. Henneicke, W. C. Preissner, and E. Christophers. 1990. IL-1 alpha or tumor necrosis factor-alpha stimulate release of three NAP-1/IL-8 related neutrophil chemotactic proteins in human dermal fibroblasts. J. Immunol. 144:2223-2232.

19. Jutila, M. A., T. K. Kishimoto, and E. C. Butcher. 1990. Regulation and lectin activity of the human neutrophil peripheral lymph node homing receptor. Blood. 76:178-183.

20. Jutila, M. A., T. K. Kishimoto, and M. Finken. 1991. Low-dose chymotrypsin treatment inhibits neutrophil migration into sites of inflammation in vivo: effects on Mac-1 and Mel-14 adhesion protein expression and function. Cell Immunol. 132:201-214.

21. Kishimoto, T. K., M. A. Jutila, E. L. Berg, and E. C. Butcher. 1989 Neutrophil Mac-1 and Mel-14 adhesion proteins inversely regulated by chemotactic factors. Science (Wash. DC). 245:1238-1241.

22. Hechtman, D. H., M. I. Cybulsky, H. J. Fuchs, J. B. Baker, and M. A Gimbrone. 1991. Intravascular IL-8: Inhibitor of polymorphonuclear leukocyte accumulation at sites of acute inflammation. J. Immunol. 147:883-892.

23. Ley, K., J. B. Baker, M. I. Cybulsky, M. A. Gimbrone, Jr., and F. W. Luscinskas. 1993. Intravenous Interleukin-8 inhibits granulocyte emigration from the rabbit mesenteric venules without altering $L$-selectin expression or leukocyte rolling. J. Immunol. 151:6347-6357.

24. Rot, A. 1991. Chemotactic potency of recombinant human neutrophil attractant/activation protein-1 (interleukin-8) for polymorphonuclear leukocytes of different species. Cytokine. 3:21-27.

25. Simonet, W. S., N. Bucay, S. J. Lauer, and J. M. Taylor. 1993. A fardownstream hepatocyte-specific control region directs expression of the linked human apolipoprotein $\mathrm{E}$ and $\mathrm{C}-\mathrm{I}$ genes in transgenic mice. J. Biol. Chem. 268:8221-8229.

26. Brinster, R. L., H. Y. Chen, M. E. Trumbauer, M. K. Yagle, and R. D. Palmiter. 1985. Factors affecting the efficiency of introducing foreign DNA into mice by microinjecting eggs. Proc. Natl. Acad. Sci. USA. 82:4438-4442.

27. MacDonald, R. J., G. H. Swift, A. E. Przybyla, and J. M. Chirgwin. 1987 Isolation of RNA using guanidinium salts. Methods Enzymol. 152:219-227.

28. Ogden, R. C., and D. A. Adams. 1987. Elecrophoresis in agarose and acrylamide gels. Methods Enzymol. 152:61-87.

29. Lauer, S. J., D. Walker, N. A. Elshourbagy, C. A. Reardon, B. LevyWilson, and J. M. Taylor. 1988. Two copies of the human apolipoprotein C-I gene are linked closely to the apolipoprotein E gene. J. Biol. Chem. 263:72777286.

30. Cohn, S. M., T. C. Simon, K. A. Roth, E. H. Birkenmeier, and J. I. Gordon. 1992. Use of transgenic mice to map cis-acting elements in the intestinal fatty acid binding protein gene (Fabpi) that control its cell lineage-specific and regional patterns of expression along the duodenal-colonic and crypt-villus axes of the gut epithelium. J. Cell. Biol. 119:27-44.

31. Sweetser, D. A., S. M. Hauft, P. C. Hoppe, E. H. Birkenmeier, and J. I. Gordon. 1988. Transgenic mice containing intestinal fatty acid-binding proteinhuman growth hormone fusion genes exhibit correct regional and cell-specific expression of the reporter gene in their small intestine. Proc. Natl. Acad. Sci. USA. 85:9611-9615. 
32. Choi, T., M. Huang, C. Gorman, and R. Jaenisch. 1991. A generic intron increases gene expression in transgenic mice. Mol. Cell. Biol. 11:3070 3074.

33. Palmiter, R. D., E. P. Sandgren, M. R. Avarbock, D. D. Allen, and R. L. Brinster. 1991. Heterologous introns can enhance expression of transgenes in mice. Proc. Natl. Acad. Sci. USA. 88:478-482.

34. Simonet, W. S., N. Bucay, S. J. Lauer, D. O. Wirak, M. E. Stevens, K. H. Weisgraber, R. E. Pitas, and J. M. Taylor. 1990. In the absence of a downstream element, the apolipoprotein $\mathrm{E}$ gene is expressed at high levels in the kidneys of transgenic mice. J. Biol. Chem. 265:10809-10812.

35. Van Zee, K. J., E. Fischer, A. S. Hawes, C. A. Hebert, T. G. Terrell J. B. Baker, S. F. Lowry, and L. L. Moldawer. 1992. Effects of intravenous IL 8 administration in nonhuman primates. J. Immunol. 148:1746-1752.

36. Von Andrian, U. H., J. D. Chambers, L. M. McEvoy, R. F. Bargatze, K.-E. Arfors, and E. C. Butcher. 1991. Two-step model of leukocyte-endothelial cell interaction in inflammation: distinct roles for LECAM-1 and the leukocyte $\beta 2$ integrins in vivo. Proc. Natl. Acad. Sci. USA. 88:538-7542.

37. Watson, S. R., C. Fennie, and L. A. Lasky. 1991. Neutrophil influx into an inflammatory site inhibited by a soluble homing receptor-IgG chimaera. Nature (Lond.). 349:164-167.

38. Jutila, M. A., L. Rott, E. L. Berg, and E. C. Butcher. 1989. Function and regulation of the neutrophil Mel-14 antigen in vivo: comparison with LFA-1 and Mac-1. J. Immuanol. 143:3318-3324.

39. Westlin, W. F., J. M. Kiely, and M. A. Gimbrone. 1992. Interleukin-8 induces changes in human neutrophil actin conformation and distribution: relationship to inhibition of adhesion to cytokine-activated endothelium. J. Leukocyte Biol. 52:43-51.

40. Mayadas, T. N., R. C. Johnson, H. Rayburn, R. O. Hynes, and D. D. Wagner. 1993. Leukocyte rolling and extravasation are severely compromised in $P$ selectin-deficient mice. Cell. 74:541-554. 